\title{
Effects of different kinds of semantic processing on memory for words
}

\author{
JANET L. PACKMAN and WILLIAM F. BATTIG \\ University of Colorado, Boulder, Colorado 80309
}

\begin{abstract}
In an incidental learning paradigm, recall and recognition memory were shown to be significantly better for words rated on pleasantness than on any of the other six semantic dimensions (concreteness, imagery, categorizability, meaningfulness, familiarity, and number of attributes) recently used for scaling of 2,854 words by Toglia and Battig (1978). Pleasantness ratings are also relatively uncorrelated with ratings on these other six dimensions, and the pattern of memory differences between these seven dimensions corresponds closely to differences in dimensional distinctiveness, as indexed by the average correlation of each dimension with the other six dimensions as reported by Toglia and Battig (1978). Word subsets with high and low mean ratings on all seven dimensions showed comparable dimensional differences in memory, but high words were both recalled and recognized better than were low words.
\end{abstract}

Several recent experiments have demonstrated quite convincingly that free recall and/or recognition performance for individual words is much improved following allegedly deeper semantic processing, as compared with nonsemantic processing conditions (e.g., Craik \& Tulving, 1975; Jenkins, 1974). Considerably less attention, however, has been directed toward the examination of possible differences between various types of semantic processing with respect to subsequent memory performance.

While there exists some recent evidence for memory differences between different semantic processing tasks or dimensions, such comparisons have shown little consistency across experiments and have typically represented only secondary considerations within experiments done primarily for other reasons. Nonetheless, there have been several demonstrations of superior memory for words processed for pleasantness or other affective properties, as compared with a variety of other semantic processing dimensions. More specifically, pleasantness processing has been shown to lead to better word memory than processing for frequency (Postman \& Kruesi, 1977), for imagery (Paivio, 1975), for concreteness (Thieman, Note 1), and for categorizability and number of attributes as well as for imagery and concreteness (Battig \& Weiss, Note 2). Klein and Saltz (1976), however, reported no consistent superiority of memory following pleasant/unpleasant ratings as compared either with the closely related happy/sad dimension or the less related fast/slow and big/little dimensions. Moreover, Shaughnessy (1976,

This is Publication 79 of the Institute for the Study of Intellectual Behavior, University of Colorado, and is based on research conducted during the first author's undergraduate internship from Wheaton College under the direction of the second author. The research was partially supported by Grant BNS 72-02084 from the National Science Foundation.
Experiment 3) found no significant differences betweer pleasantness, imagery, and frequency processing conditions. Thus even the memory superiority producec by pleasantness processing over other types of semantic processing has not been consistently demonstrated.

The present experiment was designed to provide a more direct and comprehensive comparison of free recall and recognition memory following processing on the seven semantic dimensions of concreteness (CON), imagery (IMG), categorizability (CAT), meaning. fulness (MNG), familiarity (FAM), number of attributes (NOA), and pleasantness (PLS). These are the seven dimensions on which 2,854 different words were scaled recently in the Colorado laboratories (Toglia \& Battig 1978). To avoid possible interactive effects produced through the concurrent use of multiple semantic dimensions as a basis for these ratings, each subjec1 rated all words for only one of the seven dimensions

The Toglia-Battig (1978) norms were also used tc select equal numbers of words representing two differen 1 levels (high and low) of scale values across all seven of these dimensions, thereby permitting a direct evaluation of possible differences between different word classes in relative memory performance across the seven semantic dimensions. An even more important use of the Toglia-Battig (1978) normative information involved the evaluation of memory performance as a function of the degree to which semantic ratings on a given dimension were correlated with ratings on the other six dimensions. As the results will show, there is a stron $\varepsilon$ indication that memory varies directly with the exten1 to which the semantic processing dimension is unique or unrelated to other semantic dimensions, thereby extending the recently emphasized importance of distinctiveness of encodings of individual items for effective memory (see Cermak \& Craik, 1978; Fisher \& Craik, 1977; Moscovitch \& Craik. 1976) to apply also 
to distinctiveness of the processing dimensions used for these encodings.

\section{METHOD}

\section{Subjects and Design}

All 105 subjects were introductory psychology students culfilling a course requirement option through their participation, each serving for a single session of about $40 \mathrm{~min}$. Subjects were run in small groups of 2-18. Equal-sized groups of 15 subjects used each of the seven Toglia-Battig (1978) dimensions for their word ratings. All of these dimensions, however, were used concurrently for different subjects within the same group session, through the usage of different instruction sheets.

\section{Procedure}

Each subject was first given the instructions used by Toglia and Battig (1978) for the assigned one of the seven semantic dimensions. These instructions followed basically the same format for each dimension, including detailed verbal descriptions of the dimension along with sample words illustrating the extremes of the dimension, and also emphasizing that the ratings were to be based only on that specified dimension rather than any other ways in which the words might differ. Subjects were also initially given a response sheet for the actual word ratings.

The 50 words to be rated by all subjects were then presented by slide projector for 5 sec each, each word being preceded by the 5-sec presentation of a slide containing the number from 1 to 50 representing the position of the word in the presentation sequence as designated on the subject's rating sheet. The rating sheets contained 50 rows of numbers from 1 (very low) to 7 (very high), and subjects were instructed to circle the one of the seven numbers that represented their rating for the presented word.

After all words had been rated and the rating sheets turned in, all subjects performed for $5 \mathrm{~min}$ on a nonverbal filler task that consisted of 16 sets of four similar rectangular arrays of six $x$ and o symbols. For each of these 16 items, subjects were instructed to circle the one array that they judged to be most different from the other three.

Immediately after completion of the filler task, all subjects were given a surprise written free recall test for the 50 words that they previously had rated, with a time limit of $5 \mathrm{~min}$ Subjects were instructed to write any words remembered together on the same line of the recall sheet, as suggested by Buschke (1977). Recall was followed immediately by a recognition test in which 100 words were presented in alphabetical order, including all 50 rated words intermixed with 50 distractors. All distractors were selected and judged by the authors to be semantically and/or structurally similar to one or more of the rated target words. For each word, subjects were asked to circle one of the following four symbols: $Y$ (quite sure the word was rated), YG (unsure, but best guess is that the word was rated), NG (unsure, but best guess is that the word was not rated), or $\mathbf{N}$ (quite sure the word was not rated).

\section{Materials}

All subjects rated exactly the same 50 words in the same presentation order. Equal halves of these words represented relatively high and low rating values across all of the seven dimensions. This was accomplished by selecting 25 words from two of the eight homogeneous clusters in which the word ratings are presented by Toglia and Battig (1978). More specifically, Cluster 8 consists of words with uniformly high ratings on all seven dimensions, whereas Cluster 4 contains words with average or below average ratings on each dimension. Thus the mean rating differences between the 25 high and 25 low words ranged from 2.42 for concreteness down to .51 for familiarity, with high/low differences for the other five dimensions (ordered from largest to smallest) being categorizability (2.05), pleasantness (1.85), imagery (1.71), number of attributes (.74), and meaningfulness (.56). These $25 \mathrm{high}$ and 25 low words were unsystematically intermixed in the rating presentation order, such that no more than 4 words of either type were ever presented in succession.

\section{RESULTS}

Presented in Table 1 separately for each of the seven dimensions are mean percentages of rated words that were correctly recalled (first row) and correctly recognized with $\mathrm{Y}$ responses (second row), along with mean $\mathrm{d}_{\mathrm{a}}$ measures (Simpson \& Fitter, 1973) from a signaldetectability analysis performed on the recognition data (third row). Also presented in the last row of Table 1 are the means of the six correlation coefficients for each dimension with the other six dimensions as reported by Toglia and Battig (1978, Table 1), which will become important in interpreting the present results.

\section{Memory Performance}

Since the recall measure showed the most significant differences between the seven dimensions $[\mathrm{F}(6,98)=3.58, \mathrm{MSe}=30.82, \mathrm{p}<.005]$, dimensions are ordered from low to high recall performance in Table 1. Duncan tests on the recall data showed that pleasantness was significantly superior to all other dimensions, but there were no significant differences between any of these six dimensions. Moreover, the superiority of pleasantness over the other six dimensions accounted for $75.5 \%$ of the total between-groups variance.

The recognition $d_{a}$ measure showed a closely comparable pattern of differences between the seven dimensions [overall $\mathrm{F}(6,98)=3.46, \mathrm{MSe}=.53, \mathrm{p}<.005$ ] Again only pleasantness differed significantly from each of the other six dimensions by Duncan test. This difference accounted for an even larger percentage of the total between-groups variance $(81.2 \%)$ than was found for the recall measure.

Table 1

Mean Percent Words Recalled and Recognized, $d_{a}$ Measures, and Correlations with Other Dimensions for Each of Seven Dimensions

\begin{tabular}{|c|c|c|c|c|c|c|c|}
\hline \multirow[b]{2}{*}{ Measure } & \multicolumn{7}{|c|}{ Dimension } \\
\hline & MNG & CAT & IMG & NOA & $\mathrm{CON}$ & FAM & PLS \\
\hline Percent Recall & 14.8 & 15.3 & 17.7 & 18.7 & 18.9 & 20.0 & 26.4 \\
\hline Percent Recognition & 83.5 & 78.5 & 81.6 & 86.5 & 83.2 & 79.9 & 92.8 \\
\hline Recognition $\mathrm{d}_{\mathrm{a}}$ & 2.42 & 2.01 & 2.38 & 2.48 & 2.38 & 2.34 & 3.17 \\
\hline Mean $\mathbf{r}$ & .595 & .612 & .638 & .524 & .519 & .501 & .288 \\
\hline
\end{tabular}


As for percentage of target words correctly recognized, Table 1 indicates the magnitude of differences between the seven dimensions to be closely comparable to the recall differences. However, this percent recognition measure also produced an error variance over twice as large as that for recall [overall $F(6,98)=2.74$, $\mathrm{MSe}=65.26, \mathrm{p}<.02]$. Moreover, Duncan tests indicated pleasantness not to be significantly superior to number of attributes, although pleasantness did differ significantly from each of the other five dimensions, and the difference of pleasantness from the other six dimensions still accounted for $70.3 \%$ of the total between-groups variance.

Thus all three memory measures in Table 1 showed a significant superiority for pleasantness over the other dimensions, although any differences between the other six dimensions were much smaller and nonsignificant. Analyses of false alarms (distractors given "yes" responses during recognition) also yielded similar results, and therefore are not presented.

To determine whether the foregoing memory differences might reflect greater recall organization of words rated for pleasantness than the other semantic dimensions, further analyses were performed on the small numbers of cases (only $2 \%$ ) where two words were recalled together on the same line of the recall sheet, and on adjusted-ratio-of-clustering scores (e.g., Roenker, Thompson, \& Brown, 1971) based on recall of pairs of high or of low words on adjacent lines of the recall sheet. Neither organization measure showed any evidence of differences between groups, or any significant overall organization. Groups also did not differ in recall intrusions.

\section{Memory as Related to Dimensional Relatedness}

The superiority of all measures for pleasantness over the other six dimensions, combined with the fact that pleasantness ratings show the smallest correlations with other semantic dimensions (Toglia \& Battig, 1978), suggests that the extent to which a semantic dimension is interrelated with the other types of semantic ratings may be an important determinant of memory performance. In an attempt to evaluate this possibility, the correlations between mean word ratings on all possible pairs of the seven dimensions obtained by Toglia and Battig (1978, Table 1) were used to estimate the level of interrelatedness between each one of the present dimensions and the remaining six dimensions.

These dimensional relatedness estimates were obtained by finding the mean value of the six correlation coefficients (r) obtained by Toglia and Battig (1978) between the specified dimension and each of the six other dimensions. The resulting mean correlation values are given in the last row of Table 1. Because of the questionable scale characteristics of the correlation coefficient itself, parallel comparisons were performed using both the $z$ transformations of the individual $r$ values and also the corresponding squared correlation coefficients that represent directly the proportion of variance accounted for by the correlation between the two dimensions. Since all three of these analyses yielded closely comparable results, only the results for $\mathrm{I}$ will be reported here.

Correlations were computed between the dimensional relatedness values in the last row of Table 1 and each of the recall and recognition memory measures also given in Table 1 in an attempt to obtain a quantitative estimate of the extent to which memory performance differences between the seven dimensions are associated with differences in dimensional relatedness. These correlations were all very large and negative in value, being significant at $p<.01$ for both the recall $(-.941)$ and recognition $d_{a}$ measures $(-.879)$ and falling just below the .01 level for recognition percent correct $(-.846)$, despite the fact that the seven dimensions yield only 5 degrees of freedom for these comparisons. Nonetheless, $71 \%-89 \%$ of the total variance between the present memory measures across the seven dimensions can be accounted for by differences in the extent to which their ratings are correlated with ratings on the other semantic dimensions. Since much of this relationship reflects differences of pleasantness from the other six dimensions (which also accounts for $80.1 \%$ of the variance between the seven mean $r$ values in Table 1 ), it is noteworthy that substantial negative relationships hold even when pleasantness is excluded (all $r$ values being at least -.31 and as large as -.738 for recall).

\section{Differences in Memory for High- and Low-Word Subsets}

As described in the Method section, equal halves of the present 50 words were selected to represent high and low scale values on all seven of the present dimensions, so that high and low words were evaluated as an additional factor in the percent recall and recognition analyses. Such high/low-word comparisons were not possible with the recognition $d_{a}$ measure, because distractors could not be clearly specified with respect to the high- and low-word subsets.

Table 2 contains the mean percent recall and recognition-hits measures separately for the high and low subsets for each of the seven dimensions. Overall recall performance proved to be nearly twice as great for the high- as for the low-word subsets $[F(1,98)=105.40$, $\mathrm{MSe}=16.8, \mathrm{p}<.0001]$. A closely comparable overall superiority was obtained in recognition for high over low words $[F(1,98)=78.20, \mathrm{MSe}=25.28, \mathrm{p}<.0001]$. Thus overall recall and recognition memory were markedly and comparably superior for words rated higher on the present semantic dimensions.

Of primary concern for present purposes, however, is the extent to which the superiority of pleasantness over the other six dimensions was consistent across the high and low subsets. Pleasantness showed the highest 
Table 2

Mean Percent Words Recalled and Recognized for High- and Low-Word Subsets for Each of Seven Dimensions

\begin{tabular}{|c|c|c|c|c|c|c|c|c|c|}
\hline & & \multicolumn{7}{|c|}{ Dimension } & \multirow[b]{2}{*}{ Total } \\
\hline & & MNG & CAT & IMG & $\mathrm{CON}$ & NOA & FAM & PLS & \\
\hline Recall & $\begin{array}{l}\text { High } \\
\text { Low }\end{array}$ & $\begin{array}{r}20.3 \\
9.3\end{array}$ & $\begin{array}{r}23.2 \\
7.5\end{array}$ & $\begin{array}{l}24.3 \\
11.2\end{array}$ & $\begin{array}{l}24.3 \\
13.6\end{array}$ & $\begin{array}{l}26.1 \\
11.2\end{array}$ & $\begin{array}{l}22.7 \\
17.3\end{array}$ & $\begin{array}{l}31.7 \\
21.1\end{array}$ & $\begin{array}{l}24.7 \\
13.0\end{array}$ \\
\hline Recognition & $\begin{array}{l}\text { High } \\
\text { Low }\end{array}$ & $\begin{array}{l}90.9 \\
76.0\end{array}$ & $\begin{array}{l}85.1 \\
72.0\end{array}$ & $\begin{array}{l}92.8 \\
70.4\end{array}$ & $\begin{array}{l}88.0 \\
78.4\end{array}$ & $\begin{array}{l}94.0 \\
81.1\end{array}$ & $\begin{array}{l}82.1 \\
77.6\end{array}$ & $\begin{array}{l}96.0 \\
89.6\end{array}$ & $\begin{array}{l}89.8 \\
77.9\end{array}$ \\
\hline
\end{tabular}

recall and recognition performance both for high- and low-word subsets, and this pleasantness superiority accounted for at least $56 \%$ of the total variance between the seven dimensions for all except recognition of the high-valued words. In the latter case, the $96 \%$ pleasantness recognition performance was so close to maximum that a ceiling effect may well be responsible for the reduced superiority for pleasantness over the other dimensions. In any event, this pleasantness superiority accounted for only $29.3 \%$ of the total variance between dimensions in recognition of high words.

In line with the foregoing description, there was no significant interaction of high/low words with the seven dimensions for recall $[F(6,98)=1.33, p<.25]$. This interaction was significant, however, for percent correct recognition $[\mathrm{F}(6,98)=2.75, \mathrm{MSe}=25.28, \mathrm{p}<.02]$. Further indicative of some discrepancy between the pattern of dimensional differences for recognition of high words as compared to other high and low recall and recognition measures is the fact that only for high-word recognition was there a nonsignificant correlation with the dimensional relatedness estimates given in the last row of Table 1 .

Consequently, it appears that the present recall differences between the seven dimensions are quite consistent across the high- and low-word subsets, and that recognition of low words likewise yields comparable dimensional differences. That the somewhat different pattern of dimensional differences for recognition of high words may represent a ceiling effect, and may not reflect any fundamental differences between recall/recognition and/or high/low-word types, is indicated by the results for a three-way analysis of variance including recall and recognition measures as a factor along with dimensions and high/low words. This showed no evidence for interactions of the seven dimensions with high/low-word sets, or of these two variables with recall/recognition (all Fs $<1.21, \mathrm{p}>.25$ ).

The interaction of the seven dimensions with recall/ recognition, however, was significant $[F(6,98)=2.87$, $\mathrm{MSe}=33.79, \mathrm{p}<.02]$. The latter interaction proved to be entirely attributable to the significantly smaller superiority shown in Table 1 for recognition over recall within the familiarity group (59.9) than for any of the other six groups $[F(1,98)=9.19, p<.01]$, which accounted for over half $(53.3 \%)$ of the total interaction variance. These recall/recognition differences, however, were not significantly different across the other six dimensions $[F(5,98)=1.61, p>.10]$.

\section{Subjects' Actual Ratings as Related to Recall}

An attempt was also made to evaluate any dimensional differences in memory as related to the particular ratings given to individual words, despite some serious problems in doing so. One problem is that individual subjects differed markedly in their usage of the seven rating categories, which precluded any meaningful direct comparisons across individual categories. Moreover, there is substantial evidence that individual ratings for a given word tend to be highly inconsistent and unreliable, in sharp contrast with the high reliability and stability of group mean ratings representing combinations of these unstable individual ratings (Toglia \& Battig, 1978, pp. 14-15).

Consequently, to analyze the present individual ratings data, separate linear and quadratic trend components (based upon appropriate orthogonalpolynomial coefficients) were calculated for each subject's curve representing the percent of rated items that were recalled correctly, using only the rating categories actually used by the subject. Even this modified trend analysis, however, could not be accomplished for the familiarity dimension, because most subjects never used the low-familiarity rating categories. These trend analyses were performed only on the recall data, because of the general comparability of the present recall and recognition dimensional differences, along with the complications induced by apparent recognition ceiling effects on high words rated for pleasantness as described in the preceding section. Following these trend analyses, separate comparisons across dimensions were also made for words rated at the extreme high and low ends, and also at intermediate positions, on the rating scale.

In agreement with the high/low comparisons discussed in the previous section, each of the six dimensions (excluding familiarity) showed significant $(p<.05)$ linear increases in recall from low to high individual word ratings. These linear components, however, did not differ significantly in magnitude across the six dimensions $(\mathrm{F}<1.00)$. Thus the general superiority of high over low words based upon the Toglia-Battig (1978) group ratings appears to hold also for words given higher as compared with lower ratings by individual subjects. 
Both pleasantness and number of attributes, however, also showed better recall for words given very low (1) ratings than for intermediate ratings, leading to highly significant $(p<.005)$ quadratic components only for these two dimensions that were actually larger than their significant linear components. The quadratic components for pleasantness and number of attributes were also significantly $(\mathrm{p}<.02)$ greater in magnitude than the quadratic components for the other four dimensions, which were at best marginally significant. The recall superiority for pleasantness over the other six dimensions, however, was of comparable magnitude for words given extreme and moderate individual ratings, despite the suggestion from the quadratic analysis that the pleasantness recall superiority should be greater for words given extremely high or low ratings (as found also by Thieman, Note 1).

\section{DISCUSSION}

The major finding of this experiment is the significant superiority in both recall and recognition memory produced by pleasantness processing as compared with any of the other six semantic dimensions. Since pleasantness ratings also show substantially lower correlations with ratings on the six other dimensions (Toglia \& Battig, 1978), these results suggest that the greater distinctiveness or uniqueness of pleasantness as contrasted with the other six dimensions is at least partially responsible for the present memory differences. The importance for effective memory of distinctiveness of the actual semantic as contrasted with nonsemantic encoding(s) is currently receiving heavy emphasis by levels-of-processing researchers (e.g., Cermak \& Craik, 1978; Fisher \& Craik, 1977; Klein \& Saltz, 1976; Moscovitch \& Craik, 1976).

The present results and proposed distinctiveness interpretation, however, represent an important extension beyond the distinctiveness of the particular encoding of an individual word or event, to encompass also the higher "level" of distinctiveness of the semantic processing task or dimension that is used for that encoding. More specifically, pleasantness processing appears to produce better memory because there is greater uniqueness or distinctiveness inherent in such pleasantness ratings and encodings, at least as compared with ratings on the other semantic dimensions used in this experiment. Consequently, the greater distinctiveness of pleasantness processing leads to more memorable encodings of the individual words. At a more general level, it would appear that selection of a dimension or attribute that is maximally different from other dimensions or attributes may provide an effective way of enhancing memorability.

There is also suggestive evidence from the present results that words rated by individual subjects as extremely pleasant of unpleasant are more likely to be recalled than words given medium or neutral pleasantness ratings, possibly because such neutral ratings may mean that pleasantness ratings become difficult or incongruous (Schulman, 1974). Although Thieman (Note 1) found similar memory differences favoring extreme over neutral pleasantness ratings, both his and the present results indicate superior memory following pleasantness ratings also for words given neutral ratings. However, some very recent secondary results (Weiss, Packman, \& Battig, Note 3) from an experiment primarily concerned with quite different issues do suggest that the pleasantness memory superiority can be eliminated when exclusively neutral rather than pleasant or unpleasant words are used, and when subjects have processed many words repeatedly on various combinations of three dimensions. Under the latter conditions, words rated for the two dimensions (pleasantness and number of attributes), which showed in the present study better memory following extreme than following neutral ratings, actually showed no better memory than words rated for other combinations of the Toglia-Battig (1978) semantic dimensions. The latter results contrast sharply with previous evidence (Battig \& Einstein, 1977) of superior memory when pleasantness and number-of-attributes ratings were made on words at more extreme values on these dimensions. Thus the type(s) of word(s) being rated may be an important determinant of any memory superiority for pleasantness over other types of semantic processing, with highly pleasant or unpleasant ratings representing more distinctive encodings than neutral ratings.

Of the other demonstrations of superior memory following pleasantness encoding cited in the introduction, only Postman and Kruesi (1977) offer a real alternative explanation for the superiority of pleasantness over their alternative semantic dimension of judged frequency. They characterize pleasantness as a subjective dimension, for which ratings of a particular word are based upon comparisons with previously rated words, with consequent added "displaced rehearsals" of the previously rated words. Frequency ratings, on the other hand, are presumed to represent more objective comparisons with a few specific prototypical words that are likely to be external to the list, so that frequency comparisons produce less rehearsal of previously rated words.

Most if not all of the present semantic dimensions appear more subjective than objective in the PostmanKruesi sense, and it is difficult to understand how pleasantness ratings could involve more subjective comparisons and displaced rehearsal of previous words than ratings for all of the other six dimensions (concreteness, imagery, categorizability, meaningfulness, familiarity, and number of attributes). Of these six dimensions, familiarity (which is closest to Postman and Kruesi's frequency) and number of attributes would seem to be the closest to objective dimensions with ratings based on comparisons with external standards. 
Table 1, however, shows that familiarity ranks second to pleasantness in recall performance, while number of attributes is second best on both recognition measures. So although the Postman-Kruesi interpretation may well reflect an important factor in effective memory, it clearly cannot account for the present pattern of memory differences as well as does our proposed distinctiveness interpretation.

Since pleasantness is the only one of the present seven dimensions requiring affective rather than other nonaffective types of semantic encodings, this may have some connection with the superior memorability and/or distinctiveness of pleasantness encodings. In a similar vein, Craik (Note 4) has suggested that pleasantness encodings may involve more personal reference than the other dimensions, which makes these more distinctive and memorable. Neither an affective nor a personalreference interpretation, however, is well supported by the limited research evidence presently available, since Klein and Saltz (1976) report no memory superiority for their two affective personal-reference dimensions (pleasant/unpleasant, happy/sad) as compared with nonaffective dimensions. Thus the factors underlying the superior memory and/or greater distinctiveness produced by pleasantness encodings remain to be elucidated by future research.

Although both high- and low-word subsets were included in this experiment in order to assess the generality of memory differences between the present semantic dimensions across different classes of words, the consistent superiority for high over low words itself calls for some discussion. In addition to higher ratings on all seven semantic dimensions, the high words were over four times as large in Kučera-Francis (1967) frequency counts $(85.40)$ as the low words (20.24) $[F(1,48)=7.46$, MSe $=7,113, p<.01]$. Thus the strong recognition superiority for high over low words contrasts with the general finding of recognition superiority for low-frequency words (e.g., Gregg, 1976). It should be noted, however, that the present low words were higher in frequency than the low-frequency words typically used in such frequency comparisons. Moreover, the differences of the present high and low words along other semantic dimensions probably characterize most if not all high/low-frequency comparisons, which typically make little mention of other ways in which high- and low-frequency words are different. Finally, the present results are inconsistent with Gregg's (1976) conclusion that recall superiority for high-frequency words is limited to homogeneous lists and is not found in mixed high- and low-frequency lists like those of the present study.

Despite the consistent high/low differences found for recognition as well as for recall memory, it is noteworthy that the processing dimension (familiarity) most closely related to frequency showed a significantly smaller recall-to-recognition increment, as well as a snialler high/low-word difference in both recall and recognition, than any of the other six dimensions. This suggestion that frequency-related processing attenuates any recall/ recognition or high/low-word differences deserves further investigation and may bear some relevance to the generally inconsistent pattern of high/low-frequency differences in recent recall and recognition memory experiments (e.g., Gregg, 1976).

\section{REFERENCE NOTES}

1. Thieman, T. J. What's so deep about deep processing: $A$ critical analysis of the relation between incidental orienting tasks and retention. Unpublished manuscript, 1976.

2. Battig, W. F., \& Weiss, K. R. Distinctiveness and elaboration of processing in recognition and recall memory. Paper presented at the Psychonomic Society meetings, Washington, D.C., November 1977.

3. Weiss, K. R., Packman, J. L., \& Battig, W. F. Why do pleasantness ratings produce better memory than other semantic dimensions? Paper presented at the Rocky Mountain Psychological Association meetings, April 1978.

4. Craik, F. I. M. Personal communication, February 7, 1978.

\section{REFERENCES}

Battig, W. F., \& Einstein, G. O. Evidence that broader processing facilitates delayed retention. Bulletin of the Psychonomic Society, 1977, 10, 28-30.

BuscrKe, H. Two-dimensional recall: Immediate identification of clusters in episodic and semantic memory. Journal of Verbal Learning and Verbal Behavior, 1977, 16, 201-215.

Cermak, L. S., \& CraIK, F. I. M. (Eds.). Levels of processing and human memory. Hillsdale, N.J: Lawrence Erlbaum, 1978.

Craik, F. I. M., \& Tutving, E. Depth of processing and the retention of words in episodic memory. Joumal of Experimental Psychology: General, 1975, 104, 268-294.

FisheR, R. P., \& CraIK, F. I. M. Interaction between encoding and retrieval operations in cued recall. Journal of Experimental Psychology: Human Learning and Memory, 1977, 3, 701-711.

GREGG, V. Word frequency, recognition, and recall. In $\mathbf{J}$. Brown (Ed.), Recall and recognition. New York: Wiley, 1976.

JENKINS, J. J. Can we have a theory of meaningful memory? In R. L. Solso (Ed.), Theories in cognitive psychology: The Loyola symposium. Potomac, Md: Lawrence Erlbaum, 1974.

Klein, K., \& Saltz. E. Specifying the mechanisms in a levels-of-processing approach to memory. Journal of Experimental Psychology: Human Learning and Memory, 1976, 2, 671-679.

Kučera, H., \& Francis, W. N. Computational analysis of present-day American English. Providence, R.I: Brown University Press, 1967.

Moscovitch, M., \& CRAIK, F. I. M. Depth of processing, retrieval cues, and uniqueness of encoding as factors in recall. Journal of Verbal Learning and Verbal Behavior, 1976, 15, 447-458.

Palvio, A. Coding distinctions and repetition effects in memory. In G. H. Bower (Ed.), The psychology of learning and motivation (Vol. 9). New York: Academic Press, 1975.

Postman, L., \& Kruesi, E. The influence of orienting tasks on the encoding and recall of words. Journal of Verbal Learning and Verbal Behavior, 1977, 16. 353-369.

Roenker, D. L., Thompson, C. P., \& Brown, S. C. A comparison of measures for the estimation of clustering in free recall. Psychological Bulletin, 1971, 76, 45-48. 
Schulman, A. I. Memory for words recently classified. Memory \& Cognition, 1974, 2, 47-52.

Shaughnessy, J. J. Persistence of the spacing effect in free recall under varying incidental learning conditions. Memory \& Cognition, 1976, 4, 369-377.

Simpson. A. J., \& FitTer, M. J. What is the best index of detectability. Psychological Bulletin, 1973, 80, 481-488.
Toglia, M. P., \& Batrig, W. F. Handbook of semantic word norms. Hillsdale, N.J: Lawrence Erlbaum, 1978.

(Received for publication March 1, 1978; revision accepted June 29,1978 .) 